\title{
Songbirds Conserved Sites and Intron Size of MHC Class I Molecules Reveal a Unique Evolution in Vertebrates
}

\author{
A. Arnaiz-Villena*, V. Ruiz-del-Valle, P. Reche, P. Gomez-Prieto, E. Lowry, J. Zamora, C. Areces, \\ D. Rey, C. Parga and J.I. Serrano-Vela
}

Department of Immunology, University Complutense, the Madrid Regional Blood Center, Madrid, Spain

\begin{abstract}
Birds are considered dinosaurs that passed the 65 million years ago bottleneck. Songbirds (Passeriformes) include about half extant bird species (about 5000) and are generally the most air-thriving bird species, concordantly with their small size. Mayor Histocompatibility complex (MHC) molecules stimulate immune responses against microbes and its class I molecules have seven conserved residues in all vertebrates from jawed-fishes, 300 million years ago, to humans, including chickens.

All wild songbird species tested by us $(n=18)$ and others $(n=2)$ differ in $\alpha 1$ domain residue 10 and $\alpha 2$ residue 96 from all other vertebrates. Amplification, cloning and sequencing were performed by standard methods. Sequences alignment were done by using PAUP and MEGA programs software. Crystallographic studies were performed by using mammal and bird MHC molecules from MPID database and other sources and showed that these changes did not significantly vary the MHC class I molecule stability in songbirds.

Further $\alpha 1$ and $\alpha 2$ domain comparisons by simple Composition Distances and Bayesian Inference showed that songbirds overall MHC class I molecules are phylogenetically more separated from mammal than other birds molecules. In addition MHC class I introns from Passeriformes (songbirds) were found to be longer than humans, chicken introns being the shortest ones.

These small mainly air-borne dinosaurs (Passeriformes) have undergone a different evolutive pathway, regarding to MHC, than all other tested vertebrates and more terrestrial birds. This may have been originated by an altogether different dinosaurs linage origin or to adaptation to more aerial than terrestrial environment or other unknown cause. In any case, the specific changes observed in this work for class I molecules in songbirds have reached a entropic, stable solution similar to that reached by other vertebrates.
\end{abstract}

Keywords: Songbirds, MHC evolution, immunology, passerines, carduelis, serinus.

\section{INTRODUCTION}

The Major Histocompatibility Complex (MHC) comprises the most polymorphic loci in vertebrates and its molecules present antigenic peptides to clonotypic $\mathrm{T}$ cell receptor in order to start an immune response [1]. These proteins evolve rapidly and are quite different in primary sequence of different species [2]. However, certain aminoacid residues are conserved in MHC class I molecules from reptilian to humans for keeping the overall tertiary structure [3].

MHC class I genes have been completely sequenced $(\alpha 1$ and $\alpha 2$ protein domains [1]) and thoroughly studied in Gallus gallus (chicken) $[4,5]$, and mammals $[1,6]$. The MHC locus [7] was first defined in chicken [8, 9], in particular the highly consanguineous variety 'Leghorn' [4, 10]. The chicken's MHC genetic region is considerably smaller than that of mammals - remarkably it has shorter introns [4, 11] - , and is organized quite differently [4]. Thus, a noteworthy difference is the existence of short

*Address correspondence to this author at the Departamento de Inmunología, Facultad de Medicina, Universidad Complutense de Madrid, Avenida Complutense s/n, 28040 Madrid, Spain; Tel: +34 913017354; Fax: +34 913017356; E-mail: aarnaiz@med.ucm.es introns in the chicken; that supported the hypothesis that the chicken's MHC represented a 'minimal essential MHC' [4, 5]. It has been assumed that chicken (order Galliformes) MHC was similar to all species included in the whole class Aves. However, it was shown later that this was not the case (see below).

Class I MHC genes have been sequenced and studied in four songbird species: Acrocephalus arundinaceus (great reed warbler) [12], Serinus canaria (wild canary), Serinus mozambicus (yellow-fronted canary), and Serinus thibetanus (Tibetan serin) $[13,14]$. These sequences showed an apparently more variable exon 3 than G. gallus [12-14], and that class I MHC evolution in islands was different than in sister continental species -wild canary (Serinus canaria) vs. Asian Tibetan serin and African yellow-fronted canary[14].

On the other hand, mammals and dinosaurs both appeared in Triassic Epoch — about 300 million years ago (MYA) - , and both survived the 65 MYA Cretaceous extinction. Currently there are about 4200 mammal species, and 9600 dinosaur (bird) species $[15,16]$. After the extinction, when all terrestrial dinosaurs disappeared, many mammals occupied these terrestrial niches recently left empty. 
However, mammals could not compete with aerial dinosaurs (birds) [15].

We have studied class I MHC genes and their corresponding proteins in songbirds, and compared them to other birds, and to the rest of available vertebrate genes. We focused on those domains interacting with T-cell receptors and antigen peptides and subjected to variability by balancing selection ( $\alpha 1$ and $\alpha 2$ domains) [1]. Particularly we were interested on highly conserved positions which are kept unchanged throughout vertebrate history [17]; some of them are related to interaction with the antigen $[18,19]$ or between the rest of heavy and $\beta 2$-microglobulin chains [17]. We show that some of such "universal" conserved positions throughout vertebrate evolution bear an exception to their universality in the case of songbirds. This is relevant in an evolutionary context since birds are dinosaurs that survived the Cretaceous/Tertiary extinction about 65 MYA, but are now undergoing a new extinction mainly due to human intervention. Their habitat's disappearance and pesticide spreading are deleterious for them, among other factors [15]. In the last 2000 years $20 \%$ or more aerial dinosaur species have disappeared by man-bound interventions [15] and about $50 \%$ of the extant species are currently being reduced by the same cause [20].

Air thriving tiny dinosaurs, mainly birds of order Passeriformes (songbirds) may have undergone different evolutive pressures (mainly pathogen-driven [1]) at the
MHC class I level than other vertebrates, which may include more terrestrial birds. In the present paper we have aimed to compare this key immune molecule (class I MHC) conserved sites, intron size and tertiary structure between terrestrial and air-thriving vertebrates, (mostly songbirds) to gather clues about immune system evolution and draw functional MHC conclusions in terrestrial and air-thriving vertebrates.

\section{MATERIAL AND METHODS}

\section{Sampling and Sequencing}

Class I Major Histocompatibility Complex (MHC-I) exons 2, 3, intron 2- gene sequences from 44 taxa were analyzed. Several fish, amphibians, reptiles, non-songbird aves, songbird aves, non-primate mammals, and primates were studied, as detailed in Table 1. Distribution and GenBank sequence accession numbers are given. In the case of our own samples (wild songbirds: Fringilla coelebs, and species from genera Carduelis and Serinus, $n=18$ ), blood from living birds was drawn after photographing by cutting the nail of legs locally anaesthetized with lidocaine ointment. Blood was collected in EDTA at $4{ }^{\circ} \mathrm{C}$ and frozen until use. DNA was obtained, and exons 2,3 , and intron 2 of the MHC gene were amplified with primers 3'-GTTCTCCACTCC CTGGATTACC-5' (direct) and 5'-GCGCTCCAGCTCCTT CTGCCCRTA-3' (inverse), and subsequently cloned an sequenced, [12-14, 21, 22].

Table 1. Vertebrate Species Studied and GenBank Accession Number. -: Gap or Unknown. *: Predicted Sequence. Dark Orange: Passeriformes; Light Orange: Other Birds; Dark Blue: Primates; Light Blue: Other Mammals; Green: Reptiles; White: Fishes. Songbird Species Thriving in A (Asia), E (Europe), F (Africa), NA (North America), SA (South America), P (Pacific) [43, 44]. Note that Jawed Fish, like Zebra Fish or Carp, First Appeared on Earth Over 300 MYA

\begin{tabular}{|c|c|c|c|c|}
\hline \multirow{2}{*}{ English Name } & \multirow[t]{2}{*}{ Name } & \multirow[t]{2}{*}{ GenBank \# } & \multicolumn{2}{|c|}{ Position } \\
\hline & & & 10 & 96 \\
\hline Zebra fish & Danio rerio & AAF20179 & - & Gln \\
\hline Carp & Cyprinus carpio & [3] & Thr & Gln \\
\hline African clawed frog & Xenopus laevis & {$[3]$} & Thr & Gln \\
\hline Snake & Nerodia sipedon & [3] & - & - \\
\hline Cow & Bos taurus $\mathrm{x}$ Bos indicus & ABW70136 & Thr & Gln \\
\hline Dog & Canis familiaris & NP_001014767 & Thr & Gln \\
\hline Horse & Equus caballus & NP_001075976 & Thr & Gln \\
\hline Mouse & Mus musculus & AAY85367 & Thr & Gln \\
\hline Greater Horseshoe Bat & Rhinolophus ferrumequinum & ACC68844* & Thr & Gln \\
\hline Pig & Sus scrofa & ACA33862 & Thr & Gln \\
\hline Short-beaked echidna & Tachyglossus aculeatus & AAM54212 & - & Gln \\
\hline Common gibbon & Hylobates lar & AAB08074 & Thr & Gln \\
\hline Orangutan & Pongo pygmaeus & AAK67485 & Thr & Gln \\
\hline Western gorilla & Gorilla gorilla & CAA43100 & Thr & Gln \\
\hline Chimpanzee & Pan troglodytes & BAC78189 & Thr & Gln \\
\hline
\end{tabular}


(Table 1) Contd.....

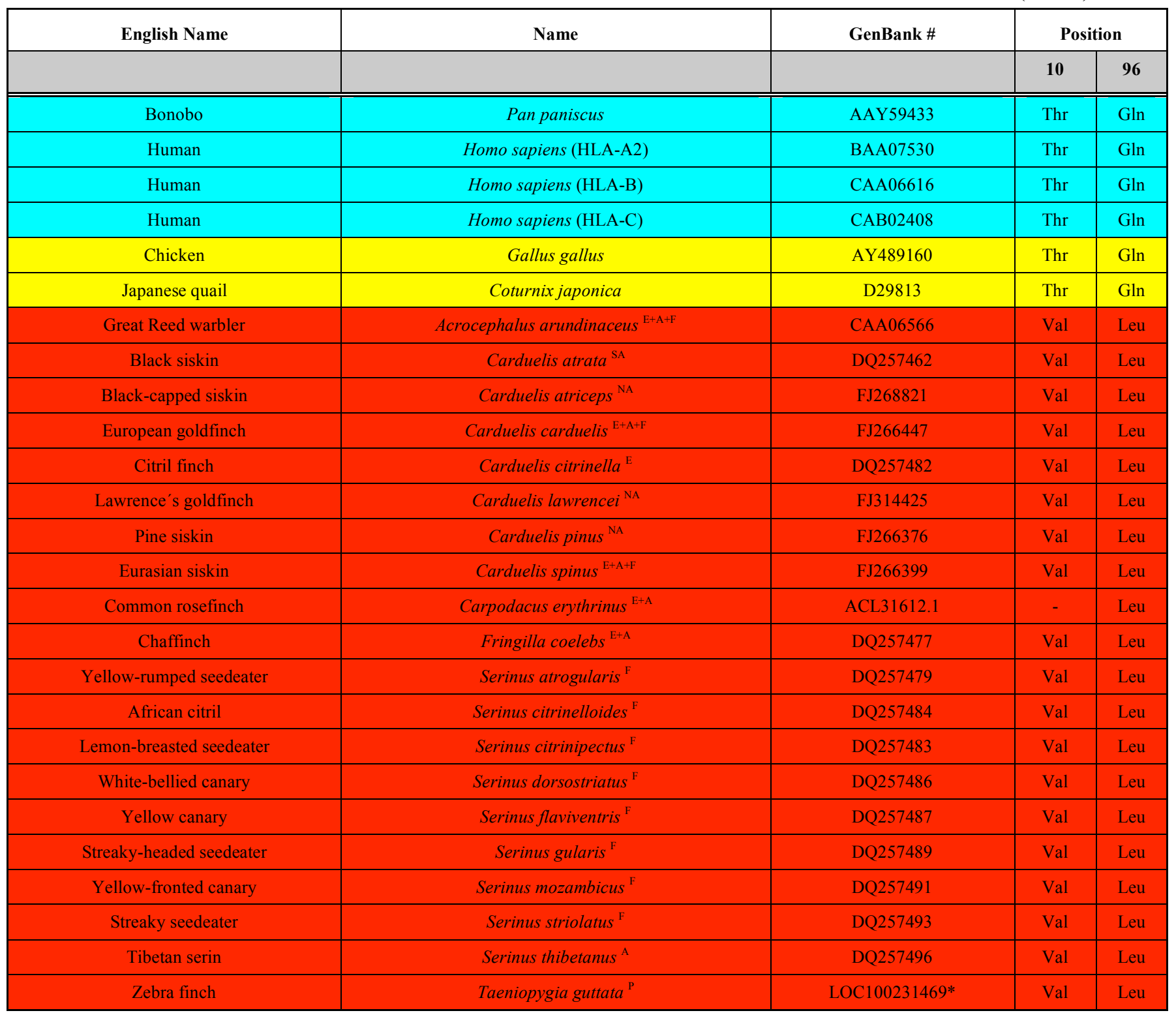

\section{Sequence Alignment and Statistical Analysis}

A 311-bp MHC sequence and a 180-aminoacid MHC sequence from vertebrate species, including Passeriformes, (Table 1, Fig. 1) were analyzed; taxa with very incomplete sequences were not included in the phylogenetic analysis in order to avoid distortion of results. Sequences correspond to MHC-I $\alpha 1$ and $\alpha 2$ domains, which conform the antigenbinding groove and the TCR-binding sites (Figs. 1 and 2). Average homology and composition distance was calculated for each species. Homology was calculated as $100 \mathrm{x}$ number of coincidences / number of known positions (i.e. excluding unknown ones and gaps). Alignment of consensus sequences was done using PAUP* [23] and MEGA software [24]. Sequences were numbered after human HLA-A2 [25].

Composition distance is a measure of the difference in nucleotide (or amino acid) composition for a given pair of sequences [26]. It is one half the sum of squared difference in counts of bases (or residues). MEGA 4 computes and presents the Composition Distance per site, which is given by the total composition distance between two sequences divided by the number of positions compared, excluding gaps and missing data (complete deletion option).

Numerical results are expressed as arithmetical mean \pm SEM (n), being $n$ the number of samples, and SEM the Standard Error of the Mean $=$ standard deviation $/ \sqrt{ } n$.

3D protein structures were first obtained and studied using Swiss Model (Deep View) [27].

Structural Analyses of Classical MHC-I Molecules (Fig. 2)

Three-dimensional (3D) structures analyzed in this study consisted of 186 murine and human classical MHC-I molecules -collected from the MPID [28] database- and a 
chicken classical MHC-I molecule (PDB:3BVA). Contacts and hydrogen bonds between $\mathrm{B} 2 \mathrm{M}$ and $\alpha$-chain residues in 3D-structures were obtained using the contact application implemented in the CCP4 suite [29] and the program "hbond" from the JOY package [30], respectively. Residues were considered to be in contact if they were at a distance of $0.4 \mathrm{~nm}$ or less. All contacts were subject to visual inspection using the program RASMOL [31].

A molecular model of the classical MHCI molecule of $T$. guttata was built by homology modelling from the 3-D coordinates of the chicken MHC class I molecule BF $2 * 2101$ (PDB: 3BVA) using the package MODELLER [32].
Accession number of the B2M and MHC-I $\alpha$-chain from $T$. guttata used for modelling were XP_002192833 and XP_002186567, respectively.

\section{Phylogenetic Analyses (Fig. 3)}

Linearized Maximum Likelihood phylogram trees were constructed with PAUP* [23] and Phylip for Windows [33]. Linearized Bayesian Inference phylograms were constructed with MrBayes [34,35], using the following settings (see [33]): outgroup $=$ Cyprinus carpio; model $=4$ by 4 , nucleotide substitution $=6$, rates $=$ invariable gamma distribution; $10^{6}$ generations; 'burn-in' $=25 \%$ of samples. Trees were

\begin{tabular}{|c|c|c|c|c|c|c|c|c|c|c|c|c|c|c|c|c|c|c|c|c|c|c|c|c|c|c|c|c|c|c|c|c|c|c|c|c|}
\hline \multirow{2}{*}{ a } & \multicolumn{36}{|c|}{ MHC-1-alpha 1} \\
\hline & 1 & 2 & 3 & 4 & 5 & 6 & 7 & 8 & 9 & 10 & 11 & 12 & 13 & 14 & 15 & 16 & 17 & 18 & 19 & 20 & 21 & 22 & 23 & 24 & 25 & 26 & 27 & 28 & 29 & 30 & 31 & 32 & 33 & 34 & 35 & 36 \\
\hline HLA-A2 & Gly & Ser & His & Ser & Met & Arg & Tyr & Phe & Phe & $\mathrm{Phr}$ & Ser & Val & Ser & $\operatorname{Arg}$ & Pro & Gly & $\operatorname{Arg}$ & Gly & Glu & Pro & Arg & Phe & Ile & Ala & Val & Gly & Tyr & Val & Asp & Asp & $\mathrm{Thr}$ & $\mathrm{Gln}$ & Phe & Val & $\operatorname{Arg}$ & Phe \\
\hline Danio_rerio & - & - & - & - & - & - & - & - & - & - & - & - & - & - & - & - & - & - & - & - & - & - & - & - & - & - & - & - & - & Val & Asp & Gly & Glu & Gln & Phe & Tyr \\
\hline Xenopus_laevi & & . & & . & Leu & & Asn & Tyr & Tyr & . & Ala & & & Asp & Arg & Ala & Phe & & Leu & & Glu & & Tyr & & Ala & . & . & r. & . & & & Leu & Ile & & & Tyr \\
\hline Amieva_amieva & - & . & & . & Leu & Gln & & 8 & Tyr & & Gly & . & & Glu & 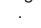 & & Glu & & Leu & & Glu & . & 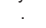 & Val & & 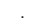 & . & & & Gly & Gln & Leu & 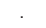 & . & Gln & Tyr \\
\hline Bos & - & . & . & . & & . & . & & Ser & & Ala & . & . & . & & . & Phe & . & & . & . & Tyr & Leu & Glu & & & . & & . & & & & . & . & Gln & \\
\hline Canis & . & . & . & . & Leu & 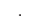 & . & . & Tyr & . & . & & . & . & & . & . & & Asp & . & . & & . & & . & . & . & & 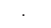 & . & . & . & . & . & . & · \\
\hline Mus & - & - & - & - & - & - & - & . & Val & . & Ala & . & & & . & . & Leu & . & & . & & Tyr & Met & Glu & . & 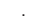 & & . & . & & . & Glu & & & & . \\
\hline 75 Sus_scrofa & - & Pro & . & . & Leu & Ser & 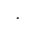 & . & Tyr & . & Ala & . & . & . & . & Asp & 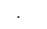 & . & Asp & Ser & . & & . & . & . & . & & . & & . & . & . & . & . & . & . \\
\hline 94 Pan_troglodytes & 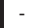 & - & - & - & - & - & - & . & & . & . & . & . & . & & . & . & . & & & & 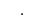 & . & . & . & . & & & & . & $\cdot$ & . & . & . & . & \\
\hline Cotur & - & - & - & - & - & - & - & & Gln & . & Ala & Met & Thr & Asp & & & Por & . & Leu & & Trp & . & Tyr & Glu & & & . & & & Gly & Glu & Ile & . & . & His & Tyr \\
\hline Gallus & - & Tyr & Pro & Ala & Val & His & Pro & & Gln & . & Ala & Met & Thr & Asp & & & Por & & Glu & 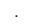 & Trp & . & Val & Thr & . & & & & . & Gly & Glu & Leu & . & . & His & Tyr \\
\hline usarundiace & Val & Leu & & . & Leu & His & & Leu & Ser & Val & Gly & . & & Glu & . & Ser & Por & & lle & 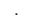 & Gln & 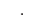 & Met & Glu & Met & & Phe & & . & Gly & lle & Pro & . & . & & Tyr \\
\hline 36 Carduelis_atrata & - & - & - & - & - & - & - & Leu & Ser & Val & Ala & . & . & Glu & & Ser & Por & & Val & . & Gln & . & Met & Ser & Thr & & & Leu & & Gly & \|le & Pro & 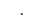 & & . & Tyr \\
\hline 33 Cardu & - & - & - & - & - & - & - & - & Thr & Val & Ala & & . & Glu & Ala & Ser & Por & . & Val & - & Gln & & Met & Ser & Thr & & . & Leu & . & Gly & \|le & Pro & & Thr & His & Tyr \\
\hline 6 Carc & - & - & - & - & - & - & - & Leu & Thr & Val & Ala & . & & Glu & 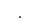 & Ser & Por & . & Val & & Gln & & Met & Ser & Thr & & . & Leu & & Gly & $\| \mathrm{e}$ & Pro & & & & Tyr \\
\hline 37 Cardu & - & - & - & - & - & - & - & - & Thr & Val & Ala & & . & Glu & & Ser & Por & & Val & . & Gln & . & Met & Ser & Met & & & Leu & & Gly & Ile & $\operatorname{Pr}$ & . & & & Tyr \\
\hline 37 Carduelis_spinus & - & - & - & - & - & - & - & - & Thr & Val & Ala & . & 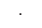 & Glu & & Ser & Por & & Val & . & Gln & . & Met & Ser & Thr & & & Leu & & Gly & Ile & Pro & . & . & & Tyr \\
\hline Frir & - & - & - & - & - & - & - & Leu & Thr & Val & Val & . & & Glu & & Ser & Por & & Val & & Lys & . & Met & Ser & Ile & & & Leu & & Gly & \|le & Pro & & - & & Tyr \\
\hline $\mathrm{Se}$ & - & - & - & - & - & - & - & Leu & Thr & Val & Val & . & 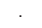 & Glu & & Ser & Por & & Val & & Gln & & Met & Ser & $\mathrm{le}$ & & & Leu & & Gly & Phe & $P$ & & . & & Tyr \\
\hline 8 Seri & - & - & - & - & - & - & - & Leu & Thr & Val & Ala & & & Glu & & Ser & Por & & Val & & Gln & . & Met & Ser & Thr & & & Leu & & Gly & $\| \mathrm{e}$ & Pro & . & . & . & Tyr \\
\hline Taeniopygia_guttata & 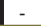 & Leu & 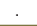 & & Leu & His & & Leu & His & Val & Ala & & . & Glu & & Ser & Por & & Val & & Gln & & Thr & Ser & Ile & & Phe & & & Gly & Ile & Pro & . & . & & Tyr \\
\hline
\end{tabular}

\begin{tabular}{|c|c|c|c|c|c|c|c|c|c|c|c|c|c|c|c|c|c|c|c|c|c|c|c|c|c|c|c|c|c|c|c|c|c|c|c|c|}
\hline & 37 & 38 & 39 & 40 & 41 & 42 & 43 & 44 & 45 & 46 & 47 & 48 & 49 & 50 & 51 & 52 & 53 & 54 & 55 & 56 & 57 & 58 & 59 & 60 & 61 & 62 & 63 & 64 & 65 & 66 & 67 & 68 & 69 & 70 & 71 & 72 \\
\hline LA-A2 & Asp & Ser & Asp & Ala & Ala & Ser & Gln & Arg & Met & Glu & Pro & Arg & Ala & Pro & Trp & Ile & Glu & Gln & Glu & Gly & Pro & Glu & Tyr & Trp & Asp & Gly & Glu & Thr & Arg & Lys & Val & Lys & Ala & His & Ser & $\mathrm{Gln}$ \\
\hline nio_rerio & yr & Tyr & . & Ser & Asn & Lys & Met & Lys & Ser & Val & 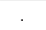 & Lys & Thr & Glu & & . & Arg & . & - & Asn & Glu & Gly & & & & Arg & $\mathrm{Gn}$ & . & Gln & Leu & Ala & lle & Gly & Tyr & His & \\
\hline & Ser & . & 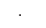 & Lys & Asp & $\mathrm{Arg}$ & Val & Glu & Ala & Ala & Thr & Gln & Trp & Met & Lys & Asp & Lys & - & 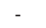 & 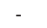 & Ala & Gly & & & Glu & $\mathrm{Gn}$ & $\mathrm{Gn}$ & Lvs & & Glu & Met & & Gly & Thr & Glu & Pro \\
\hline nieva_a & & & Asn & Thr & Arg & - & - & Glu & & Len & . & & Val & Ser & & & Lys & Asp & - & Asn & Glu & Asp & & & Glu & & Gn & & Gln & Asn & Leu & Gln & Gly & Ala & Glu & Pro \\
\hline s_t & & & . & & Pro & Asn & Pro & & & & . & . & & $\mathrm{Arg}$ & & Val & . & , & . & & & & & & & $\operatorname{Arg}$ & Asn & & & Asn & Ala & & Gly & Asn & Ala & \\
\hline anis_f & & . & . & & & Thr & Gly & & . & & & 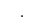 & . & & & Met & & & & 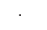 & 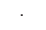 & & 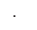 & & & Arg & . & & & Thr & & & Glu & Thr & Ala & \\
\hline us $\mathrm{r}$ & & 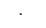 & . & & Glu & Asn & Pro & & Tyr & . & . & . & & $\mathrm{Arg}$ & & Met & & - & - & Gln & Glu & Gly & & & Glu & $\operatorname{Arg}$ & . & . & Gln & & Ala & & Gly & Asn & Glu & \\
\hline IS_. & & . & . & . & Pro & Asn & Pro & . & . & . & . & . & . & & & . & Gln & - & - & Gln & Glu & Gly & . & & . & $\mathrm{Arg}$ & & & & & Gln & $\operatorname{Arg}$ & Asp & Thr & & \\
\hline$a n$ & & . & . & 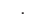 & . & & . & . & . & 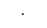 & & . & . & & & . & . & - & - & Gln & Glu & Gly & & & & Gln & . & . & & Ser & Ala & & & . & & \\
\hline oturni & & & Thr & Thr & $\operatorname{Arg}$ & - & - & . & Asn & Val & . & . & Thr & Glu & & & Lys & Ala & Gly & Ala & Val & Asp & & & Glu & $\operatorname{Arg}$ & Asn & 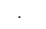 & Gln & $\| \mathrm{e}$ & & $\mathrm{Gln}$ & $\operatorname{Arg}$ & Asn & Glu & \\
\hline Gallus & Asn & . & Thr & & Val & $\operatorname{Arg}$ & $\operatorname{Arg}$ & & Tyr & Val & 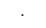 & 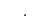 & Thr & Glu & & 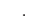 & Ala & Ala & 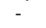 & Lys & Ala & Asp & . & & & . & $\mathrm{Gn}$ & . & Gln & Ile & Gty & Gln & Gty & Asn & Glu & \\
\hline croce & & & Glu & $\operatorname{Arg}$ & Gly & - & - & & 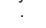 & & 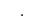 & Leu & $\mathrm{Thr}$ & Glu & & & Lys & Asp & Ala & Asp & & Gly & & & & $\operatorname{Arg}$ & Asn & & Gln & Asn & Ala & Val & Gty & Ser & Glu & His \\
\hline ardu & & & Glu & Arg & Gly & - & - & Gln & Lys & Val & . & Leu & Thr & $\mathrm{Arg}$ & & . & Lys & Asp & Ala & Glu & . & Gly & & & Glu & Arg & $\mathrm{Gn}$ & & Sin & Ile & Cys & Glu & Gty & Trp & Arg & His \\
\hline & & & Glu & Arg & Gly & - & - & Gln & Lys & Val & . & Leu & Thr & Gln & & . & Lys & Asp & Ala & Glu & & Gly & . & & Glu & Arg & Gn & & Gln & Ile & Cys & Glu & Gty & Trp & $\mathrm{Arg}$ & His \\
\hline$n c$ & & . & Glu & Arg & Gly & - & - & Gln & Lys & Val & . & Leu & Thr & Gln & & . & Lys & Asp & Ala & Glu & . & Gly & & & Glu & $\mathrm{Arg}$ & Gn & . & Sin & lle & ys & Glu & Gty & Trp & $\operatorname{Arg}$ & His \\
\hline ardu & & & Glu & Arg & Gly & - & - & Gln & Lys & Val & & Leu & Thr & $\mathrm{Arg}$ & & 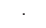 & Lys & Asp & Ala & Glu & . & Gly & & & Glu & Arg & $\mathrm{Gn}$ & & Gln & Ile & Cys & Glu & Gty & $\operatorname{Trp}$ & $\operatorname{Arg}$ & His \\
\hline & & . & Glu & Arg & Gly & - & - & Gln & Lys & Val & & Leu & Thr & Arg & & & Lys & Asp & Ala & Glu & & Gly & & & u & Arg & $\mathrm{Gn}$ & . & In & Ile & ys & & Gty & & Arg & His \\
\hline Fingilla & & . & Glu & Arg & Gly & - & - & & Lys & Val & & Leu & Thr & Gln & & Met & & Asp & - & & Ala & & & & Glu & $\operatorname{Arg}$ & & & Gln & Thr & Cys & Gln & Gty & Trp & $\operatorname{Trp}$ & His \\
\hline & & 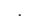 & Glu & Arg & Gly & - & - & Gln & Lys & Val & . & Leu & Thr & Gln & & Met & & Ala & 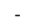 & & Val & & & & Glu & $\operatorname{Arg}$ & Gn & & In & Ile & Cys & Glu & Gty & Trp & Arg & His \\
\hline & & 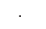 & Glu & Arg & Gly & - & - & Gln & Lys & Val & & Leu & Thr & Gln & & . & Lys & Asp & - & & Ala & . & & & Glu & Arg & Gn & & in & Ile & Cys & Glu & Gty & Trp & $\operatorname{Arg}$ & His \\
\hline Taeniopygia_guttata & & & Glu & Arg & Gly & 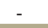 & - & & Ala & & . & Leu & Thr & Gln & & Met & Lys & Asp & - & . & Ala & & & & & Gn & & & Gln & lle & Ser & Val & Arg & Asn & Gln & His \\
\hline
\end{tabular}

\begin{tabular}{|c|c|c|c|c|c|c|c|c|c|c|c|c|c|c|c|c|c|c|}
\hline & 73 & 74 & 75 & 76 & 77 & 78 & 79 & 80 & 81 & 82 & 83 & 84 & 85 & 86 & 87 & 88 & 89 & 90 \\
\hline HLA-A2 & Thr & His & Arg & Val & Asp & Leu & Gly & Thr & Leu & Arg & Gly & Tyr & Tyr & Asn & Gln & Ser & Glu & Ala \\
\hline Danio rerio & Val & Tyr & Lys & & & Phe & Gln & . & & Lys & Glu & $\operatorname{Arg}$ & Phe & . & & . & $\mathrm{Gln}$ & - \\
\hline Xenopus_laevi & Val & Phe & Lys & His & Asn & Val & Lys & . & Ala & Met & Glu & Arg & Phe & . & & . & Thr & - \\
\hline Amieva_amieva & Val & Phe & & Gly & Asn & $\mathrm{He}$ & Asn & & Ala & Met & Asn & $\operatorname{Arg}$ & & . & . & Thr & Gly & - \\
\hline Bos_taurus & Ser & Phe & . & , & Asn & & Asn & . & & & . & & . & & . & . & . & . \\
\hline Canis_familiaris & Arg & Tyr & & & & . & Asp & . & & & . & & & . & . & . & . & \\
\hline Mus musculus & Ser & Phe & & & . & & $\operatorname{Arg}$ & & & Leu & & & 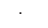 & . & & . & Lys & Gly \\
\hline Sus_scrofa & & Tyr & . & & Gly & . & Lys & Asn & & 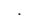 & . & & & . & . & . & 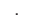 & \\
\hline Pan_troglodytes & & Asp & & & . & . & & . & · & . & . & & & . & - & . & . & Asp \\
\hline Coturnixjaponica & Asn & Ser & . & & Ser & & Asp & Asn & Val & Ala & $\operatorname{Arg}$ & Leu & . & . & & & Gly & $r$ \\
\hline Gallus_gallus & Ile & Asp & . & Glu & Asn & . & & $\| \mathrm{le}$ & & Gln & $\operatorname{Arg}$ & $\operatorname{Arg}$ & . & & . & Thr & Gly & - \\
\hline Acrocephalusarundiaceus & Val & Thr & Ala & $\operatorname{Arg}$ & Asn & . & Glu & $\| \mathrm{le}$ & & . & Glu & Arg & . & . & 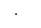 & . & Gly & - \\
\hline Carduelis_atrata & Val & Glu & Ala & Arg & Asn & & Glu & & 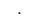 & Gln & Asp & $\operatorname{Arg}$ & . & . & & . & Gly & - \\
\hline Carduelis_carduelis & Val & Glu & Ala & Arg & Asn & . & Glu & . & . & Gln & Asp & $\operatorname{Arg}$ & & . & . & . & Gly & - \\
\hline Carduelis_lawrencei & Val & Glu & Ala & Arg & Asn & & Glu & & & Gln & Asp & $\operatorname{Arg}$ & & & & & Gly & - \\
\hline Carduelis_pinus & Val & Glu & Ala & Arg & Asn & . & Glu & . & . & Gln & Asp & $\operatorname{Arg}$ & & . & & & Gly & - \\
\hline Carduelis_spinus & Val & Glu & Ala & Arg & Asn & 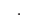 & Glu & . & . & $\mathrm{Gln}$ & Asp & $\operatorname{Arg}$ & & . & & . & Gly & - \\
\hline Fringilla_coelebs & Val & Glu & Ala & Arg & Asn & & Glu & . & . & Gln & . & $\operatorname{Trp}$ & . & & & Thr & Gly & - \\
\hline Serinus_atrogularis & Val & Glu & Ala & Arg & Asn & . & Glu & & & Gln & Asp & $\operatorname{Arg}$ & & . & & & Gly & 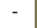 \\
\hline Serinus_thibetanus & Val & Glu & Ala & Arg & Asn & . & Glu & . & . & Gln & Asp & Arg & & & & & Gly & - \\
\hline Taeniopygia_guttata & Val & Asp & Ala & $\operatorname{Arg}$ & Asn & . & Glu & . & 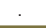 & $\mathrm{Gln}$ & Glu & Arg & 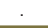 & . & & . & Arg & - \\
\hline
\end{tabular}


(Fig. 1) Contd.....

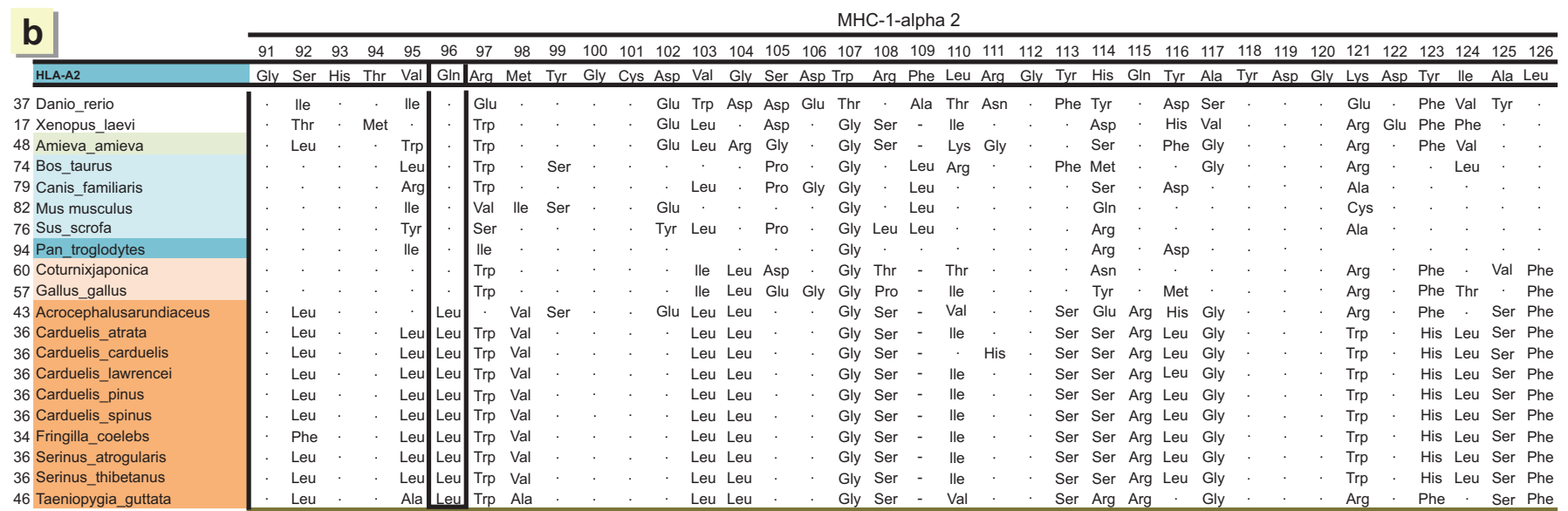

\begin{tabular}{|c|c|c|c|c|c|c|c|c|c|c|c|c|c|c|c|c|c|c|c|c|c|c|c|c|c|c|c|c|c|c|c|c|c|c|c|c|}
\hline & 127 & 128 & 129 & 130 & 131 & 132 & 133 & 134 & 135 & 136 & 137 & 138 & 139 & 140 & 141 & 142 & 143 & 144 & 145 & 146 & 147 & 148 & 149 & 150 & 151 & 152 & 153 & 154 & 155 & 158 & 157 & 158 & 159 & 160 & 161 & 162 \\
\hline LA-A2 & Lys & Glu & Asp & Leu & Arg & Ser & Trp & Thr & Ala & Ala & Asp N & Met & Ala & Ala & Gln & Thr & Thr & Lys & His & Lys T & & Glu & Ala & Ala & His & Val & Ala & Glu & Gln & Leu & Arg & Ala & Tyr & Leu & Glu & - \\
\hline nio re & D & Thr & Glu & Glu & Trp & Val & Tyr & Val & Pro & Ser & Val & Phe & Tyr & & & Leu & & Thr & Gln & . & . & sn & Pro & Glu & Val & Asn & . & & $\operatorname{Arg}$ & Asn & Lys & Asn & & . & Ser & \\
\hline & & Lys & u & Thr & Leu & Thr & & & . & & & Ser & GI & & & & & & & & & Asp & Leu & Glu & Ala & Met & Asn & Gln & Gly & $\mathrm{gg}$ & Lys & & & & & \\
\hline & & & & & & . & . & 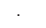 & 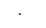 & Gly & Glu & Thr & Glu & & . & lle & & & Arg & & . & & & & Gly & Tyr & & . & Val & Gln & ? & Asn & & . & & \\
\hline & & & . & 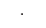 & & & & . & . & & . & Thr & $\cdot$ & . & & lle & & $\operatorname{Arg}$ & Arg & & & & . & & $y$ & Thr & & & His & Asp & & Asn & & & & \\
\hline & & & & & Lys & Thr & & & . & . & . & . & & . & Leu & lle & & . & & & & 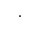 & Gln & & Gly & Glu & . & & $\operatorname{Arg}$ & & . & & . & & & \\
\hline & & . & & 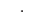 & . & 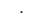 & . & & & . & $T$ & Thr & 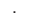 & . & & Ile & & & $\operatorname{Arg}$ & & 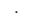 & 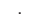 & Thr & & Asn & & & & $\operatorname{Arg}$ & $\operatorname{Arg}$ & r. & Ser & & & & \\
\hline & & & . & & . & . & . & & & & & & 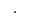 & . & & $\| \mathrm{le}$ & & & $\operatorname{Ar}$ & & . & . & & & & Ala & . & & & Gln & & & . & & Gln & \\
\hline an_- & & Lys & . & Thr & Met & Thr & Phe & & . & & Val & Pro & Glu & . & Val & Pro & & . & $\mathrm{Ar}$ & & & 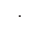 & Glu & Gly & Asp & Tyr & & 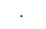 & Arg & Gln & Lys & His & & & & \\
\hline & & Lys & Gly & Thr & Met & Thr & Phe & & 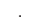 & 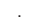 & Val & Pro & Glu & & al & Pro & & & Arg & & . & & Glu & Gly & Ser & Glu & Pro & & $g$ & Trp & Lys & Asn & 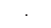 & & & \\
\hline & & Leu & c & Ser & Gly & Arg & Phe & Val & & & & Ser & & & Glu & $\| \mathrm{e}$ & & $\operatorname{Arg}$ & $\operatorname{Arg}$ & His & & . & Glu & Gly & Ile & . & & . & gg & Lys & Thr & Asn & 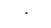 & & $\cdot$ & \\
\hline & & Pro & 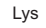 & Ser & Gly & s & Phe & Val & Pro & & Asn & Ser & $\mathrm{Se}$ & & u & 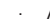 & Asn & Gly & Lys & $\mathrm{H}$ & & . & u & Gly & Ile & Glu & Val & & 9 & 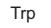 & ir & Asn & & & /s & \\
\hline & & Pro & Lys & Ser & Gly & Lys & Phe & Val & Pro & & Asn & Ser & $\mathrm{Se}$ & & 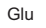 & 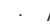 & & Gly & Lys & His & & & $\mu$ & Gly & e & Glu & Val & & $g$ & & a & Asn & & & $\mathrm{s}$ & \\
\hline & & Ser & Lys & Ser & Gly & s & Phe & Val & Pro & . & $n$ & Ser & $\mathrm{Se}$ & . & lu &. & ר & Gly & Lys & $\mathrm{H}$ & . & 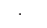 & Glu & Gly & Ile & Glu & Val & & $g$ & 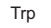 & Thr & Asn & & & $\mathrm{s}$ & \\
\hline & & Pro & Lys & Ser & Gly & Lys & Phe & Val & Pro & & Asn & Ser & $\mathrm{S}$ & & Glu & & Asn & Gly & Lys & $\mathrm{H}$ & & . & Glu & Gly & Ile & Glu & Val & & $g$ & Trp & Thr & Asn & & & s & \\
\hline & & Pro & Lys & Ser & Gly & Lys & Phe & Val & Pro & & Asn & Ser & S & & Glu & & Asn & Gly & Lys & $\mathrm{H}$ & . & & $\mu$ & Gly & Ile & Glu & Val & . & $g$ & $T$ & Thr & Asn & & & $\mathrm{s}$ & \\
\hline & & Leu & c & Ser & G & Lys & Phe & Val & $\mathrm{Pr}$ & . & Asn & Ser & $\mathrm{T}$ & & Glu & Ile & & $\operatorname{Arg}$ & Lys & $\mathrm{H}$ & & & & Gly & Ile & Glu & Gly & & & & M & A & . & & & \\
\hline & & Pro & Lys & Ser & Gly & Lys & Phe & Val & Pro & . & Asn & Ser & S & & u & & Asn & Gly & Lys & $\mathrm{H}$ & & . & $\mu$ & Gly & Ile & Glu & Val & & $\mathrm{rg}$ & Trp & 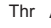 & Asn & 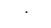 & & is & \\
\hline & & Pro & Lys & Ser & Gly & Lys & Phe & Val & Pro & & Asn & Ser & Ser & & Glu & & Asn & Gly & Lys & b & & & u & Gly & Me & Glu & Val & & $\mathrm{rg}$ & Trp & Thr & Asn & & & & \\
\hline & & Leu & Gly & Ser & Gly & Lys & Phe & & & & & S & & . & Glu & $\| \mathrm{e}$ & & $g$ & $\operatorname{Arg}$ & & . & & u & Asp $N$ & Met & - & . & & rg & & Lys & Asn & & & $\mathrm{s}$ & \\
\hline leniopygia_guttata & $\mathrm{sp}$ & Leu & Gly & Ser & Gly & Lys & Phe & Leu & 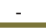 & - & & Ser & - & - & Glu & Ile & - & $\operatorname{Arg}$ & Arg & $\operatorname{Arg}$ & - & 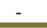 & Glu & Asp $N$ & Met & 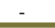 & - & & $\operatorname{Arg}$ & . & Lys & Asn & 1 & - & Lys & \\
\hline
\end{tabular}

\begin{tabular}{|c|c|c|c|c|c|c|c|c|c|c|c|c|c|c|c|c|c|c|c|c|}
\hline & 163 & 164 & 165 & 166 & 167 & 168 & 169 & 170 & 171 & 172 & 173 & 174 & 175 & 176 & 177 & 178 & 179 & 180 & 179 & 180 \\
\hline HLA-A2 & Thr & Cys & Val & Glu & Trp & Leu & Arg & Arg & Tyr & Leu & Glu & Asn & Gly & Lys & Glu & Thr & Leu & $\mathrm{Gln}$ & $\operatorname{Arg}$ & Thr \\
\hline Danio_rerio & Glu & . & lle & . & . & & Glu & Lys & . & Met & Gln & Tyr & . & & Ser & - & - & - & - & - \\
\hline Xenopus_laevi & Ile & 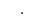 & lle & & Asp & . & Lys & Lys & & & Ser & Tyr & & Gln & - & - & - & - & - & - \\
\hline Amieva_amieva & Phe & . & Ile & . & . & . & Gln & Lys & & . & $\operatorname{Arg}$ & Tyr & . & Asn & Lys & - & - & - & - & - \\
\hline Bos_taurus & Glu & . & . & . & & . & & . & & . & & - & - & - & - & - & - & - & - & - \\
\hline Canis_familiaris & . & . & & . & 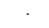 & . & . & . & & . & & Met & . & . & . & - & - & - & - & - \\
\hline Mus musculus & . & . & . & . & . & . & & & & . & Lys & - & - & - & - & - & - & - & - & - \\
\hline Sus_scrofa & Leu & . & . & & Ser & & & Glu & & . & & Met & 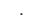 & & Asp & - & - & - & - & - \\
\hline Pan_troglodytes & & . & . & & & & ${ }^{\circ}$ & . & & & . & - & - & - & - & - & - & - & - & - \\
\hline Coturnixjaponica & . & . & . & Gln & & . & . & & His & Val & . & - & - & - & - & - & - & - & - & - \\
\hline Gallus_gallus & . & . & . & & & & . & & & Val & . & - & - & - & - & - & - & - & - & - \\
\hline Acrocephalusarundiaceus & Glu & . & Pro & . & . & . & Gln & & His & Val & $\operatorname{Arg}$ & Tyr & . & Gln & Lys & - & - & - & - & - \\
\hline Carduelis_atrata & Leu & & Pro & & Ser & . & & Lys & & Val & Gly & - & - & - & - & - & - & - & - & - \\
\hline Carduelis_carduelis & Leu & 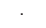 & Pro & . & Ser & . & & Lys & & Val & Gly & - & - & - & - & - & - & - & - & - \\
\hline Carduelis_lawrencei & Leu & . & Pro & . & Ser & . & . & Lys & & Val & Gly & - & - & - & - & - & - & - & - & - \\
\hline Carduelis_pinus & Leu & 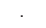 & Pro & . & Ser & & . & Lys & & Ile & Gly & - & - & - & - & - & - & - & - & - \\
\hline Carduelis_spinus & Leu & . & Pro & & Ser & & . & Lys & & Val & Gly & - & - & - & - & - & - & - & - & - \\
\hline Fringilla_coelebs & Arg & & Pro & Gly & Ser & . & Gln & Lys & & \|le & $\operatorname{Arg}$ & - & - & - & - & - & - & - & - & - \\
\hline Serinus_atrogularis & Leu & . & Pro & & Ser & . & . & Lys & & Val & Gly & - & - & - & - & - & - & - & - & - \\
\hline Serinus_thibetanus & Leu & & Pro & & Ser & . & & Lys & & Val & Gly & - & - & - & - & - & - & - & - & - \\
\hline Taeniopygia_guttata & Lys & & Pro & . & & . & & Lys & & Val & Gly & Tyr & & Gln & Lys & Glu & . & Glu & & Lys \\
\hline
\end{tabular}

Fig. (1). MHC sequences. 1a. Alpha-1 chain aminoacid sequences. 1b. Alpha-2 chain aminoacid sequences. Specifically conserved residues $(10,96)$ in class I MHC molecules in Passerine birds thriving in a world-wide range. Non-Passerine birds and other vertebrates show Thr and Gln at positions 10 and 96 (boxed), respectively, since 300 MYA, while songbirds bear Val and Leu (boxed). Numbering of positions is referred to HLA-A2 molecule [3,37,45]. Zebra finch sequence is deduced from [38]. Common rosefinch $\alpha 2$ domain sequence is taken from [39].

displayed with MEGA, treated with CorelDraw [36] for esthetic purposes, and exported to "tiff" format.

\section{RESULTS AND DISCUSSION}

\section{Songbirds Bear Val10 Instead of Thr10 at $\alpha 1$ Chain and Leu96 Instead of GIn96 at $\alpha 2$ Chain}

Seven positions in vertebrates had been shown to be conserved in MHC class I $\alpha 1$ and $\alpha 2$ protein chains from jawed fishes to humans (from Devonic Epoch, 300 MYA, until present) [3]. These conserved residues are: Thr10, Asp29, and Asn86 in the $\alpha 1$ chain, and Gln96, Gly100, Cys101, and Cys164 in the $\alpha 2$ chain. $\alpha 1$ and $\alpha 2$ domains are basically forming a valve where antigenic peptides accommodate and are presented to the $\mathrm{T}$-cell receptor in order to start an immune response [1, 37]. Conserved residues are probably under a strong evolutive pressure to maintain the class I MHC valve tri-dimensional structure 


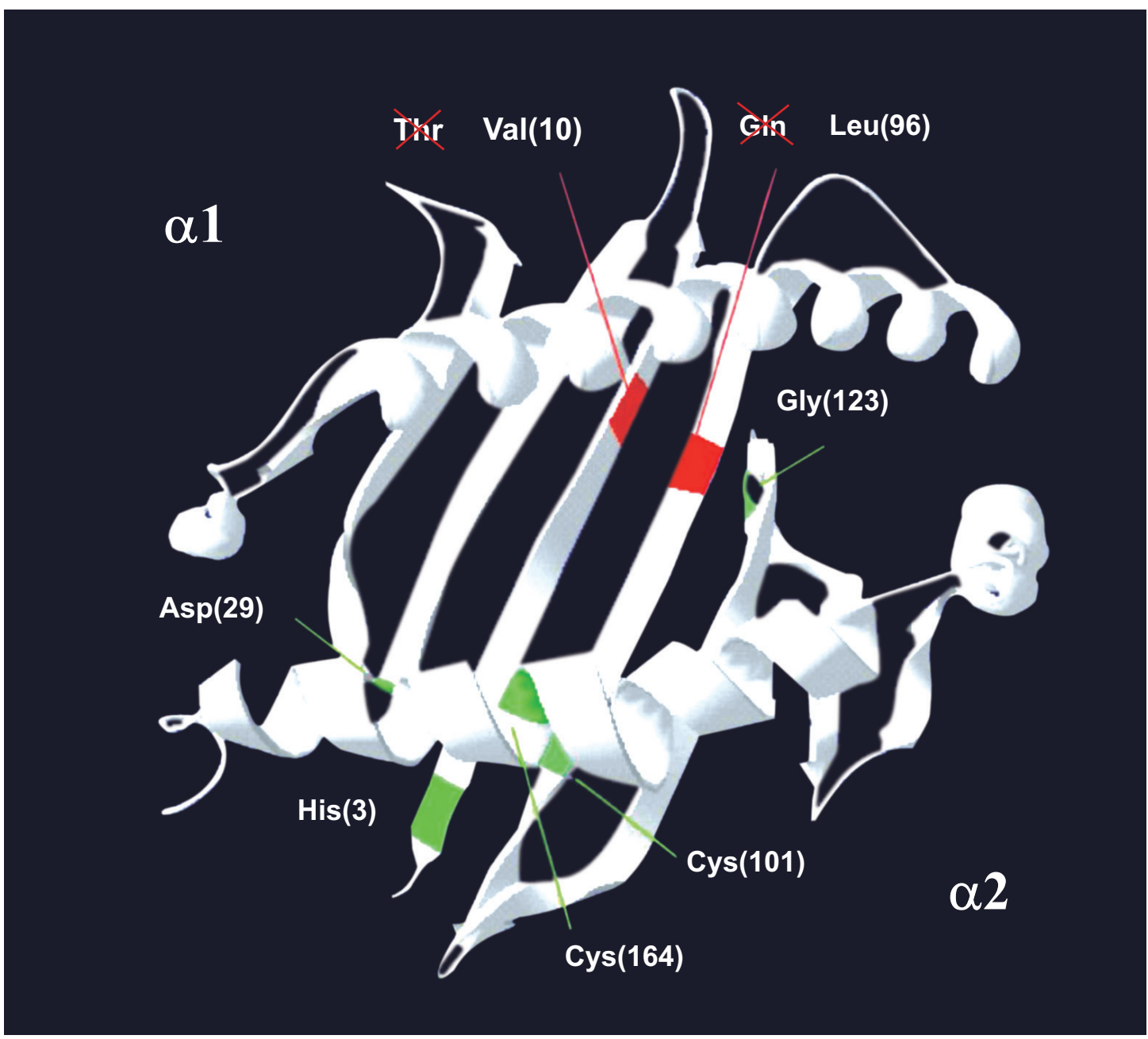

Fig. (2). The MHC-I molecule. Three-dimensional model of domains $\alpha-1$ and $\alpha-2$ of a songbird Class I histocompatibility molecule. Green: five positions conserved in vertebrates [3], including chicken and pheasant. Red: the two conserved positions (10 and 96) showing a different aminoacid in studied songbirds compared to the rest of vertebrates. In brackets: aminoacid position. See Table 1, Fig. (1).

$[3,37]$. However, some of these conserved residues at such a wide time-evolutionary scale are not kept in half of the extant birds: songbirds (Passeriformes) tested so far; they bear Val10 instead of Thr10 on $\alpha 1$ chain and Leu96 instead of Gln96 on $\alpha 2$ chain (Figs. 1 and 2, Table 1). All Passerine birds' MHC sequences, except those of warbler and zebra finch predicted sequence [38] have been obtained by us. All continents, latitudes and environments are represented with the songbird studied species (Table 1).

Songbirds preserve different MHC class I constant residues than other vertebrates, but also than other, more terrestrial birds, like chicken (G. gallus) and Japanese quail (Coturnix japonica) (Table 1, Fig. 1).

A phylogenetic classification of birds, partly based on DNA hybridization was put forward by Sibley and Alquist [16]. Our own studies have shown that Passeriformes (4600 species) of Serinus and Carduelis Genera bear these two aminoacid changes, unlike other extant bird species such as G. gallus (Table 1, Figs. 1 and 2). Also, other Passeriformes, like Taeniopygia guttata (zebra finch) MHC class I obtained from a whole genome linkage map [38] and Acrocephalus arundinaceus (great reed warbler) [12] are compared; Common rosefinch $\alpha 2$ sequence is taken from [39].
Interactions of both differential conserved songbird residues in a tridimensional $\mathrm{MHC}$ molecule are as follows:

1. Side chains of residues Thr10 and Gln96 in all vertebrates do not interact with each other or with peptide (see Methodology).

2. Side chains of both residues interact with $\beta 2$-microglobulin (B2M) and appear to contribute to the overall structure of MHC class I molecules.

3. In terrestrial vertebrate residues (including chicken and quail birds):

a. Gln96 establishes two hydrogen bonds with B2M atoms: one with His31 and another one with Trp60.

b. Trh10 is nested between residues Met54 and Phe62 of B2M; in addition, Thr10 is interacting with a trapped $\mathrm{H}_{2} \mathrm{O}$ molecule (see Methodo$\log )$.

4. Specific songbird residues (Table 1, Fig. 2)

a. Change to Leu96 will result in a loss of the two stabilizing hydrogen bonds with B2M 


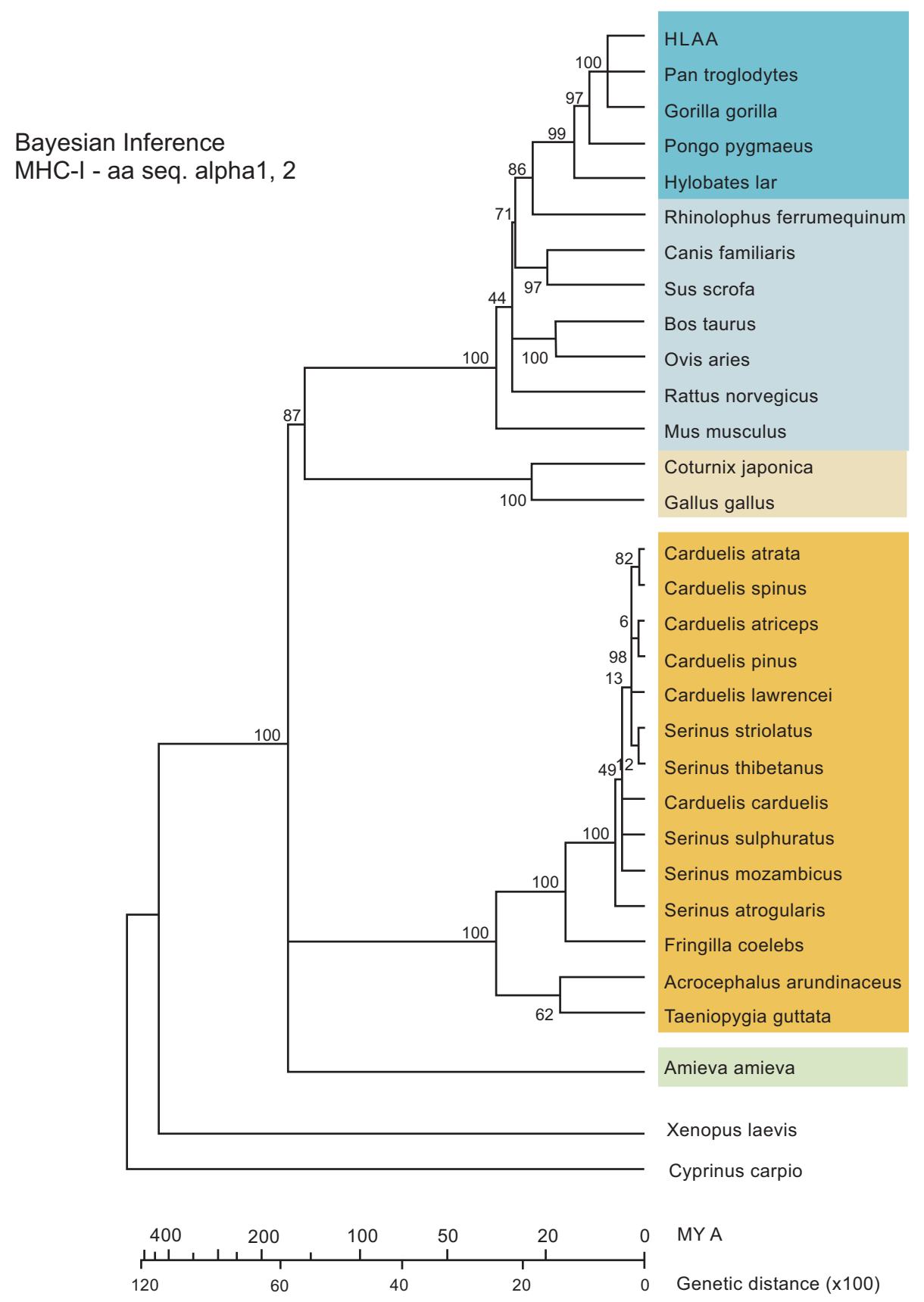

Fig. (3). Linearized Bayesian Inference phylogram. It is built from alpha-1 and alpha-2 aminoacid sequences. Dark orange: Passeriformes; light orange: other birds; dark blue: primates; light blue: other mammals; green: Amieva lizard; white: other vertebrates. Three different vertebrate groups are found: one for reptiles, one for mammals and more "terrestrial" birds, and one for more "aerial" birds. Time divergence of lineages has been found more reliable, if molecules other than selection pressured MHC ones are used. (i.e: mtDNA molecules which are thought to be subjected to constant evolutive pressures).

b. Change to Val10 may compensate the previous change regarding the B2M attachment affinity to the $\alpha$ chain, because Van der Waals interactions with B2M's Met54 and Phe62 arise; moreover, the trapped $\mathrm{H}_{2} \mathrm{O}$ molecule will be released with a subsequent entropy gain (see Methodology).

B2M contributes to stability of the quaternary structure of the groove [17]. Therefore, and assuming that B2M residues in Passerines are equivalent as the B2M in humans (which is indeed concluded because of deduced zebra finch
B2M sequence $[17,38])$, it seems that these couple of changes will also maintain solid relationships between MHC class I $\alpha$ an $\beta$ molecules in songbirds.

On the other hand (Fig. 1), sequences from songbird's $\alpha 1$ and $\alpha 2$ domains seem to be overall different from other vertebrates. Considering composition distance [24] from HLA-A2 (not shown), it is found that it is much higher to songbirds $(0.93 \pm 0.08, \mathrm{n}=11, \mathrm{p}<0.00001)$, than to other vertebrates $(0.43 \pm 0.08, \mathrm{n}=19)$ or to reptiles/fishes $(0.34 \pm 0.07$, $\mathrm{n}=16$ ). Distance from songbirds to the rest of vertebrates is 
$0.86 \pm 0.01(\mathrm{n}=220)$, whereas distance from songbirds to the rest of birds is even higher: $0.95 \pm 0.05(\mathrm{n}=22)$.

\section{Taxonomical Implications}

Sibley and Alquist [16] counted, in 1990, 9946 bird species, some of which are nowadays probably extinct [15]. Over half of them, 5875, are Passerine birds. Although MHC was discovered in Gallus gallus [9], very few bird species have been sequenced for MHC class I genes/protein valves, with the exception of those shown in Table 1. Extant species of order Galliformes have shown to be of earlier appearance on Earth than order Passeriformes, at least than families Fringillidae (Fringilla coelebs or chaffinch, genus Carduelis, genus Serinus) [21, 22] and Passeridae (Old World sparrows [40], estrildids [40, 41], Ploecinae or weavers [40]). This does not mean that Fringillidae lineage may be older; extinct Fringillidae and other bird data are lacking. A parallel but distinct evolution of Galliformes and Passerines (and other bird lineages) starting long before the 65 MYA dinosaur "extinction" may be possible. However, the fact that more than half of the extant species are songbirds, or Passeriformes, in contrast to the other extant 32 orders [16] is remarkable. The main differences that Passeriformes make are their small size in relation to other birds, and their mainly aerial environment.These small dinosaurs survivors have either passed more easily the 65 MYA bottleneck or they have been more successful in speciation diversity altogether; whatever is the cause, it is clear that bigger extant Galliformes and other more terrestrial birds seem to have appeared earlier than Passeriformes on Earth [21, 22, 40], even if evolution of their lineages started in a non-determined, much older time.

\section{Evolutionary Consequences and Bird Introns}

In addition, a Bayesian Inference dendrogram constructed with MHC class I aminoacid sequences clearly separates Galliformes from Passerines (Fig. 3), which further stresses the different evolution regarding to MHC of these two types of genera within the class Aves. Also, Passerine MHC class I introns shown in Fig. (4) do not correspond in length and homology to that of G. gallus. Songbirds have longer introns than humans, and much longer than G. gallus.

Also, homology with Gallus gallus MHC class I intron 2 sequence, which is $38.3 \%$ for HLA-A2 and $32.5 \%(n=36)$ for Passeriformes, shows that a "minimum essential MHC" [4] may only apply to chicken and not to other birds.

Only introns from genera Carduelis and Serinus have been sequenced, but the coincidence of MHC class I conserved residues in $\alpha 1$ and $\alpha 2$ domains on the vertebrate lineage in Passerine birds (genera Carduelis, Serinus, Acrocephalus, Passer) vs. non-Passerine birds (Galliformes, Gallus gallus) suggests that this may be a general intron characteristic for all Passeriformes, although more work is needed in more bird genera. Intron 2 has more base pairs (bp) in songbird's MHC class I (304 $\pm 1 \mathrm{bp}, \mathrm{n}=36)$ than in human's HLA-A (238 bp, $\mathrm{n}=82$ [25], being the chicken's intron the shortest one $(203, n=1)$. This further supports the evolutive different pathways of Passerine vs. non-Passerine birds.

Finally, it seems that the evolutionary features of extant songbirds (about half of bird species [16]) is different to mammals and other birds, regarding MHC class I genes. These are very important to start an immune response and some questions may be posed:

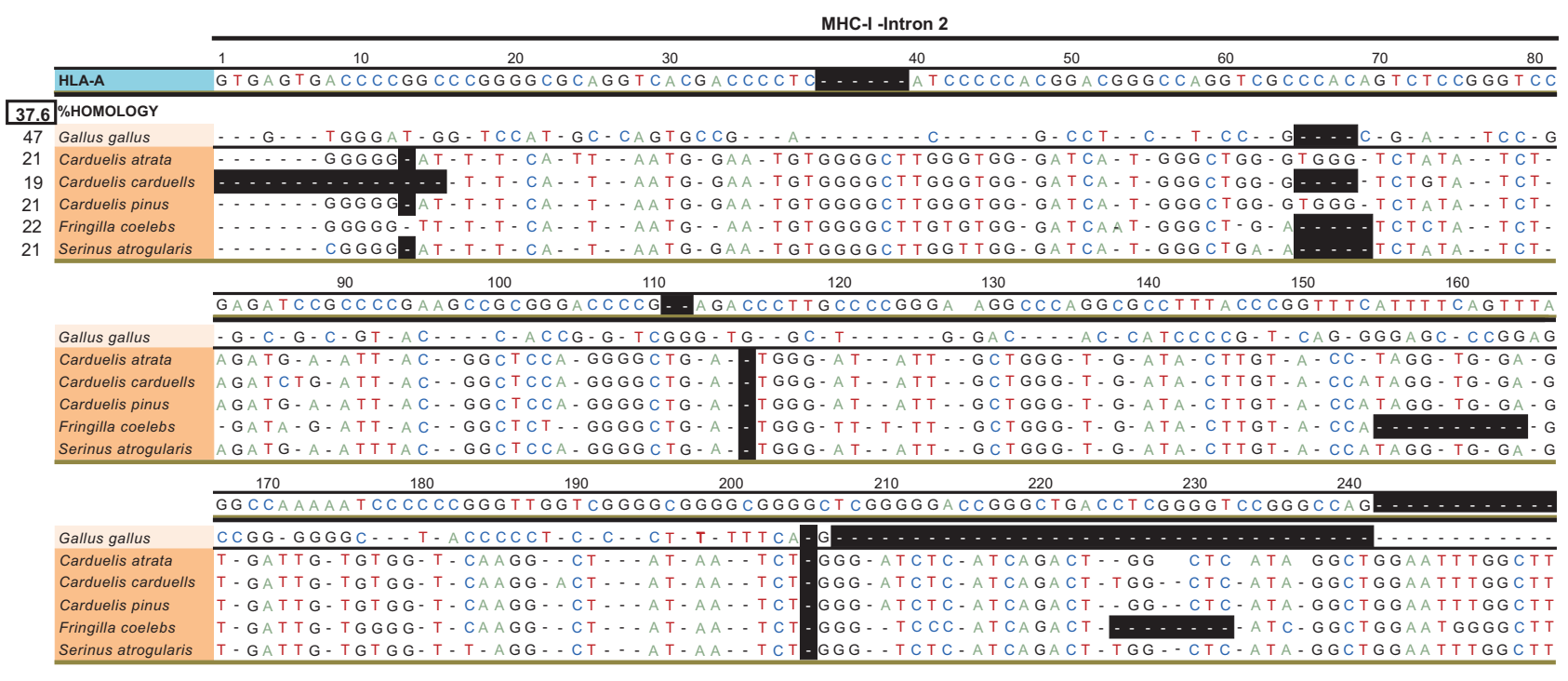

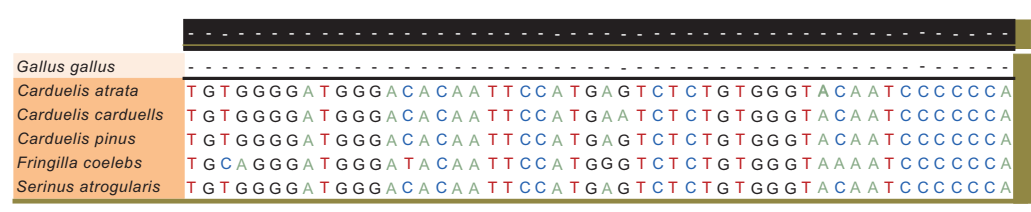

Fig. (4). Intron 2 sequences showing percentage of homology with HLA-A. Dark orange: Passeriformes; light orange: chicken; dark blue: human. 
1. Are songbirds direct descents of dinosaurs, while chicken is not, or are they descents of an altogether different dinosaur lineage? There is not sufficient dinosaur evolution details to answer this question, although non-flying dinosaurs had color feathers [42], remarking that feathers may have not evolved for flight but for mating choice or other reasons.

2. Are songbirds different to all other extant vertebrates, including other more terrestrial birds like chicken, in MHC class I molecules because they have been selected for advantages in mostly air life environment?

These questions may be answered as more data become available. However, chaffinch (Fringillinae), zebra finch (Estrildinae), great reed warbler (Sylvidae) and Carduelinae (common rosefinch, canaries, goldfinches) are relatively quite distant for justifying a generalization within Passerines, which will possibly have exceptions when more data are available.

Recently, zebra finch genome has been deduced by automated computational analysis from a predicted genomic sequence (NW_002197937) [38]. Although MHC class I genes were not specifically "sequenced", a part of genomic DNA (XP_002186567) seems to be a class I antigen (Table 1). In addition, this zebra finch outlined genome shows how different chicken chromosomes [7] are from zebra finch's: many more gene placements than expected are rearranged, or in other words, they are found in different chromosome or linkage groups. This further stresses the large difference in genetic homology between two types of birds: a galliform (chicken [16]) and a Passerine or songbird (zebra finch [16]). This is in concordance with the conserved MHC class I residues: chicken resembles other vertebrates while zebra finch resembles all available songbirds (Table 1, Figs. 1 and 2).

\section{ACKNOWLEDGEMENTS}

This work was supported in part by grants from the Spanish Ministry of Health (FISS PI051039 and PI080838), Spanish Ministry of Foreign Affairs (A/9134/07 and $\mathrm{A} / 17727 / 08$ ) and three different Mutua Madrileña Automovilista grants.

\section{ABBREVIATION}

$\mathrm{MHC}=\quad$ Major histocompatibility complex

\section{REFERENCES}

[1] Klein J. Natural history of the major histocompatibility complex. New York, USA: J. Willey and Sons 1986.

[2] Danska J, McDevitt H. Evolution and vertebrate immunity. Austin: University of Texas Press 1987.

[3] Grossberger D, Parham P. Reptilian class I major histocompatibility complex genes reveal conserved elements in class I structure. Immunogenetics 1992; 36: 166-74.

[4] Kaufman J, Volk H, Wallny HJ. A "minimal essential MHC" and an "unrecognized MHC": two extremes in selection for polymorphism. Immunol Rev 1995; 143: 63-88.

[5] Kaufman J, Jacob J, Shaw I, et al. Gene organisation determines evolution of function in the chicken MHC. Immunol Rev 1999; 167: 101-17.
[6] Bjorkman PJ, Saper MA, Samraoui B, Bennett W, Strominger J, Wiley DC. The foreign antigen binding site and $\mathrm{T}$ cell recognition regions of class I histocompatibility antigens. Nature $1987 \mathrm{~b} ; 329$ : 512-8.

[7] Briles W, Briles R. Identification of haplotypes of the chicken major histocompatibility complex (B). Immunogenetics 1982; 15: 449-59.

[8] Briles W, McGibbon W, Irwin DM. On multiple alleles affecting cellular antigens in the chicken. Genetics 1950; 35: 633-40.

[9] Briles W, McGibbon W. Heterozygosity of inbred lines of chickens of two loci effecting cellular antigens. Genetics 1948; 33(6): 605.

[10] Simonsen, Arnul M, Sorensen P. The chicken MHC and its importance. In: Zijpp A, Sybesma W, Eds. Improving genetic disease resistance in farm animals. Dordrecht: Kluwer 1989.

[11] Kroemer G, Zoorob R, Auffray C. Structure and expression of a chicken MHC class I gene. Immunogenetics 1990; 31: 405-9.

[12] Westerdahl H, Wittzell H, von Schantz T. Polymorphism and transcription of MHC class I genes in a passerine bird, the great reed warbler. Immunogenetics 1999; 49: 158-70.

[13] Arnaiz-Villena A, Moscoso J, Ruiz-del-Valle V, et al. Bayesian phylogeny of Fringillinae birds: status of the singular African oriole finch (Linurgus olivaceus) and evolution and heterogeneity of genus Carpodacus. Acta Zool Sin 2007b; 53: 826-34.

[14] Arnaiz-Villena A, Lowy E, Ruiz-del-Valle V, et al. Evolution of the major histocompatibility complex class I genes in Serinus canaria from the Canary Islands is different from that of asian and african continental Serinus sp. J Ornithol 2007a; 148: S479-S84.

[15] Sanz J. Los dinosaurios voladores: historia evolutiva de las aves primitivas. Ediciones Libertarias/Prodhufi 1999.

[16] Sibley CG, Ahlquist J. Phylogeny and classification of birds. New Haven, Conn: Yale University Press 1990.

[17] Tysoe-Calnon V, Grundy J, Perkins S. Molecular comparisions of the B2M-binding site in class I major histocompatibility complex $\alpha$-chains and proteins of related sequences. Biochem J 1991; 277: 359-69.

[18] Hashimoto K, Okamura K, Yamaguchi H, Ototake M, Nakanishi T, Kurosawa Y. Conservation and diversification of MHC class I and its related molecules in vertebrates. Immunol Rev 1999; 167: 81100 .

[19] Madden D. The three-dimensional structure of peptide-MHC complexes. Annu Rev Immunol 1995; 13: 587-622.

[20] Wilson E. The diversity of life. UK: Harvard University Press 1992.

[21] Arnaiz-Villena A, Alvarez-Tejado M, Ruiz-del-Valle V, et al. Phylogeny and rapid northern and southern hemisphere speciation of goldfinches during the Miocene and Pliocene epochs. Cell Mol Life Sci 1998; 54:1031-41.

[22] Arnaiz-Villena A, Alvarez-Tejado M, Ruiz-del-Valle V, et al. Rapid radiation of canaries (Genus Serinus). Mol Biol Evol 1999; 16: 2-11.

[23] Swofford DL. PAUP*. Phylogenetic analysis using parsimony (* and other methods) version 4. Sunderland, Massachusetts: Sinauer Associates 2002

[24] Tamura K, Dudley J, Nei M, Kumar S. MEGA4: molecular evolutionary genetics analysis (MEGA) software version 4.0. Mol Biol Evol 2007; 24: 1596-9.

[25] The Anthony Nolan Trust. The anthony nolan trust. http://www.anthonynolan.org.uk 2009.

[26] Kumar S, Gadagkar S. Disparity index: a simple statistic to measure and test the homogeneity of substitution patterns between molecular sequences. Genetics 2001; 158: 1321-7.

[27] Peitsch MC. Protein modelling by e-mail. Bio Technol 1995; 13: 658-60.

[28] Govindarajan K, Kangueane P, Tan T, Ranganathan S. MPID: MHC-Peptide interaction database for sequence-structure-function information on peptides binding to $\mathrm{MHC}$ molecules. Bioinformatics 2003; 19: 309-10.

[29] The CCP4 Suite: Programs for Protein Crystallography. Acta Crystallogr D Biol Crystallogr 1994; 50: 760-3.

[30] Mizuguchi K, Deane C, Blundell T, Johnson M, Overington J JOY: protein sequence-structure representation and analysis. Bioinformatics 1998; 14: 617-23.

[31] Sayle R, Milner-White E. RASMOL: biomolecular graphics for all. Trends Biochem Sci 1995; 20: 374.

[32] Sali A, Blundell T. Comparative protein modelling by satisfaction of spatial restraints. J Mol Biol 1993; 234: 779-815. 
[33] Felsenstein J. PHYLIP. Department of genome sciences, university of Washington, Seattle. 1-1-2010.

[34] Huelsenbeck JP, Ronquist F. MRBAYES: Bayesian inference of phylogeny. Bioinformatics 2001; 17: 754-5.

[35] Huelsenbeck JP, Larget B, van-der-Mark P, Ronquist F, Simon D. MrBayes: Bayesian inference of phylogeny. Department of Scientific Computing: Florida State Univ 2010

[36] Corel Corporation. CorelDRAW Graphics Suite X4. Corel Corp 2010.

[37] Bjorkman PJ, Saper MA, Samraoui B, Bennett WS. Structure of the human class I histocompatibility antigen, HLA-A 2. Nature 1987a; 329: 506-12.

[38] Stapley J, Birkhead T, Burke T, Slate J. A linkage map of the zebra finch Taeniopygia guttata provides new insights into avian genome evolution. Genetics 2008; 179: 651-67.

[39] Promerová M, Albrecht T, Bryja J. Extremely high MHC class I variation in a population of a long-distance migrant, the scarlet rosefinch (Carpodacus erythrinus). Immunogenetics 2009; 61: $451-61$.
[40] Allende LM, Rubio I, Ruiz-del-Valle V, et al. The old world sparrows (genus Passer) phylogeography and their relative abundance of nuclear mtDNA pseudogenes. J Mol Evol 2001; 53: 144-54.

[41] Arnaiz-Villena A, Ruiz-del-Valle V, Gomez-Prieto P, Reguera R, Parga-Lozano C, Serrano-Vela JI. Estrildinae finches (aves, passeriformes) from Africa, South Asia and Australia: a molecular phylogeographic study. Open Ornithol J 2009; 2: 29-36.

[42] Zhang F, Kearns S, Orr P, et al. Fossilized melanosomes and the colour of cretaceous dinosaurs and birds. Nature 2009; 463:1075-8.

[43] Clement P, Harris P, Davies J. Finches and sparrows. London: Croom Helm 1993.

[44] Lepage D. Avibase: The world bird database. http://avibase.bsceoc.org/species.jsp?lang=ES\&avibaseid=9820CECA12EC737D\&s ec=map 2010.

[45] Parham P, Lomen CE, Lawlor DA, et al. Nature of polymorphism in HLA-A, -B, and -C molecules. Proc Natl Acad Sci USA 1988; 85: 4005-9.

Received: July 27, 2010

Revised: October 08,2010

Accepted: October 22,2010

(C) Arnaiz-Villena et al.; Licensee Bentham Open.

This is an open access article licensed under the terms of the Creative Commons Attribution Non-Commercial License (http://creativecommons.org/licenses/by$\mathrm{nc} / 3.0 /$ ), which permits unrestricted, non-commercial use, distribution and reproduction in any medium, provided the work is properly cited. 